\title{
Self-Monitoring for Speech and its Links to Age, Cognitive Effort, Schizotypal Trait Expression and Impulsivity During Adolescence
}

George Salaminios a , Larisa Morosan ${ }^{\mathrm{b}, \mathrm{c}}$, Elodie Toffel ${ }^{\mathrm{b}}$, Michal Tanzer $^{\mathrm{a}}$, Stephan Eliez ${ }^{\mathrm{c}}$, Martin Debbané ${ }^{\mathrm{a}, \mathrm{b}, \mathrm{c}}$

${ }^{a}$ Research Department of Clinical, Educational and Health Psychology, University College London, UK, $5^{\text {th }}$ Floor, 1-19 Torrington Place, London WC1E 7HB, UK

b Developmental Clinical Psychology Unit, Faculty of Psychology, University of Geneva, Switzerland, 40 boulevard du Pont-d'Arve, CH-1211 Geneva, Switzerland

${ }^{c}$ Department of Psychiatry, Developmental Imaging and Psychopathology Lab, Office Medico-Pédagogique, University of Geneva 1 rue David-Dufour, CH-1211 Geneva, Switzerland

George Salaminios, g.salaminios@ucl.ac.uk, Research Department of Clinical, Educational and Health Psychology, University College London, UK, $5^{\text {th }}$ Floor, 1-19 Torrington Place, London WC1E 7HB, UK

Larisa Morosan, 1arisa.morosan@unige.ch, Developmental Clinical Psychology Unit, Faculty of Psychology, University of Geneva, Switzerland, 40 boulevard du Pont-d'Arve, CH-1211 Geneva, Switzerland

Elodie Toffel, elodie.toffel@unige.ch, Developmental Clinical Psychology Unit, Faculty of Psychology, University of Geneva, Switzerland, 40 boulevard du Pont-d'Arve, CH-1211 Geneva, Switzerland Michal Tanzer, ‥tanzer@ucl.ac.uk, Research Department of Clinical, Educational and Health Psychology, University College London, UK, $5^{\text {th }}$ Floor, 1-19 Torrington Place, London WC1E 7HB, UK

Stephan Eliez, stephan.eliez@unige.ch, Department of Psychiatry, Developmental Imaging and Psychopathology Lab, Office Medico-Pédagogique, University of Geneva 1 rue DavidDufour, CH-1211 Geneva, Switzerland Martin Debbané, martin.debbane@ unige.ch,Developmental Clinical Psychology Unit, Faculty of Psychology, University of Geneva, Switzerland, 40 boulevard du Pont-d'Arve, CH-1211 Geneva, Switzerland 
Corresponding author: George Salaminios, g.salaminios@ucl.ac.uk

Research Department of Clinical, Educational and Health Psychology, University College London, UK, $5^{\text {th }}$ Floor, 1-19 Torrington Place, London WC1E 7HB, UK

Tel: +44(0)2076791947, Fax: +44(0)2079168502

\begin{abstract}
Introduction: Disruptions in self-monitoring processes represent key cognitive factors associated with schizophrenia spectrum disorders. In the current study, we assessed the effects of age and cognitive effort on self-monitoring for speech in adolescence, as well as its associations with personality dimensions pertaining to schizotypy and impulsivity.

Methods: 121 community adolescents undertook a self-monitoring task that assesses the capacity to discriminate between self-generated overt and silent speech, for items requiring different levels of cognitive effort. Self-report measures were used to assess trait dimensions of schizotypy and impulsivity.
\end{abstract}

Results: Cognitive effort, but not age, contributed to the overall rate of self-monitoring errors. Contrary to clinical psychosis and high risk samples, increased cognitive effort in healthy adolescents led to more internalizing than externalizing self-monitoring errors. Higher scores on the interpersonal dimension of schizotypy were associated with increases in the total rate of self-monitoring errors. No associations were found between positive schizotypy and externalizing self-monitoring misattributions. Finally, trait impulsivity dimensions were not associated with self-monitoring performance.

Conclusions: The present findings suggest that self-monitoring confusions may be linked to trait-risk for psychosis in adolescence. Future studies can prospectively assess whether the association between negative schizotypal traits and self-monitoring represents a distal marker of psychosis vulnerability.

Keywords: source monitoring; schizotypy; schizophrenia; personality; psychosis risk 


\section{Introduction}

According to contemporary conceptualizations, clinical manifestations of psychotic illnesses, particularly those that entail cognitive-perceptual aberrations, are associated with confusions in the identification of self-generated information. For instance, auditory hallucinations have been linked with difficulties in monitoring the origin one's own inner speech (Allen et al., 2007), while delusions of control have been attributed to difficulties in monitoring the initiation and sensory consequences of self-produced actions (Blakemore et al., 2003). A key cognitive process associated with the monitoring of self-generated material is the ability to discriminate between different sources of memory information, known as source monitoring (Johnson et al., 1993).

Source-monitoring processes are typically divided into three types: (1) reality-monitoring involves the capacity to distinguish between information generated by oneself and information generated by another agent; (2) external source-monitoring refers to the ability to distinguish between two external sources of information (e.g. between words spoken by a male or female voice); and (3) self-monitoring involves the capacity to discriminate between two internal sources of information (what one imagined doing or saying from what one did or said). To study these processes, Johnson et al., (1993) developed the source-monitoring framework, a conceptual approach seeking to explain the parameters that promote accurate sourcemonitoring discriminations, as well as factors that underlie source-monitoring confusions. According to this model, judgments about the source of memory information are based on evaluations pertaining to the characteristics of the memory events themselves. These include the contextual (spatial/temporal) and sensory characteristics (visual/auditory) available, as well as the record of cognitive operations engaged during the original encoding of an event. For instance, studies suggest that memories for externally-perceived events contain more sensory information compared to memories for imagined events and the capacity to discriminate 
between the two increases with the amount and clarity of such detail contained in the former (Dewhurst, 1999; Johnson, 1988). Conversely, because imagination is less automatic than perception, memories for imagined events contain more records about the cognitive operations that led to their creation compared to those for real events (Sussman, 2001). Indeed, increased cognitive effort during memory encoding for imagined events has been shown to support accurate source-monitoring discriminations (Finke et al., 1988).

Regarding impairments in source memory, the source-monitoring framework postulates that because contextual characteristics and cognitive operations are used as cues that determine the origin of memory events, source confusions are likely to occur under conditions that alter the quantity and quality of such information during encoding (Johnson et al., 1993). For example, studies indicate that conditions that increase the sensory detail encoded for imagined events may lead to their misattribution as externally-perceived and real (Johnson, 1988). Similarly, increases in the cognitive elaboration required during the encoding of externallyperceived events may lead to their misattribution as self-generated and imagined (Johnson, 2006). Although source memory is an imperfect process and transient source confusions are relatively common, impairments in source-monitoring have also been linked with the phenomenology of psychotic illnesses.

Reality-monitoring research in psychosis has shown that patients with schizophrenia and individuals at clinical high-risk for the illness (CHR) display a tendency towards misattributing the source of self-generated items to external agents (Bentall et al., 1991; Brébion et al., 2000; Johns et al., 2010; Johns et al., 2006). This pattern of externalizing reality-monitoring misattributions is particularly pronounced among patients reporting hallucinations (Bentall et al., 1991; Brébion et al., 2000) and non-clinical adults experiencing hallucination-like phenomena (Larøi et al., 2004). Interestingly, increased cognitive effort appears to exacerbate the rate of externalizing misattributions in these samples (Bentall et al., 1991; Larøi et al., 
2004). This suggests that unlike healthy controls, schizophrenia sufferers and high-risk subjects fail to utilize cognitive effort as a cue to monitor the source of self-generated information (Larøi et al., 2004).

Given that psychotic experiences entail pervasive difficulties in discriminating between imagination and reality, studies have also investigated self-monitoring processes in schizophrenia. These have addressed whether participants can distinguish between internal sources of information (what one did from what one thought or imagined doing). For example, Keefe et al. (1999) found that together with difficulties in discriminating between selfgenerated and experimenter-produced words, patients with schizophrenia also displayed confusions in memory between words they read aloud and words they read silently. In line with reality-monitoring research, self-monitoring studies suggest that patients with schizophrenia and CHR individuals demonstrate externalizing misattributions towards recalling imagined stimuli as overtly enacted (Docherty, 2012; Franck et al., 2000; Gawęda et al., 2012; Henquet et al., 2005).

According to Docherty (2012), source discriminations for "internal" events are never purely internal. This is because once internal events are enacted, they also become part of the perceptually-based external world. For instance, contrary to silent speech, overt speech entails the generation of motor actions (i.e. vocalizations), along with the perception of the sensory feedback produced by these actions (i.e. listening to the spoken material). In the same vein, Johnson et al. (1993) indicate that discriminating in memory between covert/imagined mental events (i.e. silent speech) and events that have adopted a public quality (i.e. overt speech) may in itself constitute an instance of reality-monitoring. Therefore, the overlap between various types of source discrimination problems in psychosis may signify the presence of a more global deficit in the processing of internal and external information (Docherty, 2012). Indeed, according to the source-monitoring framework, source misattributions occur due to alterations 
in the encoding of sensory and cognitive information (Johnson et al., 1993). This is also in line with the forward model of motor control (Frith et al., 2000), which postulates that source confusions result from failures to match the intended or predicted outcomes of self-generated actions to their externally-perceived sensory consequences. This can occur either due to disruptions in generating predictions about the outcome of actions, or due to impairments in the processing of the sensory feedback related to these actions. In both cases, because of the high level of sensory discrepancy between cognitive prediction and sensory feedback, selfgenerated events are experienced as external to the self. Taken together, the source-monitoring framework and the forward model suggest that the source confusions observed in psychotic illnesses are sustained by impairments in the monitoring of sensory and cognitive information.

Another line of research indicates that source-monitoring impairments are also observable, at an attenuated level, among non-clinical individuals in the context schizotypal trait expression, prior to development of clinical symptoms (Peters et al., 2007). Schizotypal manifestations represent distal risk factors for schizophrenia (Debbané et al., 2014) and most psychometric analyses examining their factorial structure identify three dimensions: the cognitive-perceptual (positive schizotypy: hallucination, delusion-like phenomena), interpersonal (negative schizotypy: social anxiety, constricted affect), and disorganization dimensions (odd behaviours, odd speech) (Fonseca-Pedrero et al., 2018; Raine, 2006). Only a limited number of studies to date have analysed the associations between schizotypal dimensions and self-monitoring in non-clinical individuals. Peters et al. (2007) examined the relationship between action self-monitoring (distinguishing between performed physical actions versus imagined actions) and positive schizotypy in healthy adults (Mage $=21.41$ ). They found that participants reporting high positive schizotypy displayed more externalizing misattributions for actions (confusing imagined actions as overtly enacted), compared to those with lower scores. Recently, Humpston et al. (2017) found that cognitive-perceptual 
schizotypal manifestations in community adults $\left(\mathrm{M}_{\mathrm{age}}=22.30\right)$ were positively associated with the total rate of action self-monitoring errors. Although suggestive of a relationship between self-monitoring and sub-clinical signs of psychosis, these studies involve adult participants either within or beyond the critical period of illness onset. From an early intervention standpoint, important insights can be gained by examining self-monitoring during earlier developmental stages.

Adolescence represents a key period during which schizotypal manifestations become clinically relevant (Gooding et al., 2005). Debbané et al. (2010) reported that adolescents (Mage $=13.97$ ) prone to express positive schizotypal manifestations due to $22 \mathrm{q} 11.2$ deletion syndrome, a neurogenetic condition conferring high-risk for schizophrenia, exhibited more externalizing and internalizing self-monitoring speech misattributions compared to controls. Similarly to adult clinical samples, increased cognitive effort exacerbated the rate of selfmonitoring errors in adolescents with 22q11.2 deletion syndrome (Debbané et al., 2010). Despite these findings, no study to date has examined the relationship between schizotypal dimensions and disruptions in self-monitoring among typically developing adolescents. Research on adolescent schizotypy, which precedes the emergence of clinical manifestations, can increase our understanding regarding the earliest phases of self-monitoring impairments in schizophrenia, with implications for early prevention treatment.

Impulsivity represents another personality feature associated with the emergence of clinical psychopathology (Moeller et al., 2001). Impulsivity constitutes a multidimensional trait reflecting the tendency to act rashly without regard to negative consequences (Moeller et al., 2001). According to Whiteside and Lynam (2001) it is comprised of four dimensions: urgency (tendency to act rashly under positive or negative affect); lack of premeditation (tendency to not think about consequences of actions); lack of perseverance (inability to remain focused); and sensation seeking (tendency to pursue new and exciting activities). Evidence suggest that 
increased levels across impulsivity dimensions lie at the centre of multiple problematic behaviours arising in adolescence, such as antisocial behaviour, substance abuse, risk-taking and delinquency (Moeller et al., 2001). Although most psychological problems associated with impulsivity also entail cognitive difficulties (Müller et al., 2015), little is known about the relationship between source-monitoring and impulsivity. Our group recently showed that incarcerated adolescents that commonly report higher levels of impulsivity compared to community adolescents, exhibit increased rates of externalizing and decreased rates of internalizing self-monitoring misattributions (Morosan et al., 2018). Interestingly, the only study that has examined the relationship between impulsivity and schizotypy in adolescence reported inverse associations between the two (Badoud et al., 2015). Thus, self-monitoring may represent a transdiagnostic cognitive mechanism associated to different personality traits involved in the emergence of psychotic and non-psychotic manifestations (Ferchiou et al., 2010). To our knowledge, studies encompassing evaluations of impulsivity when examining self-monitoring in adolescence are currently lacking.

In the present study, we examine self-monitoring in community adolescents using a task that assesses the capacity to discriminate between self-generated overt and silent speech under different levels of cognitive effort (Debbané et al., 2010). Our main aims are to assess the effects of age and cognitive effort on self-monitoring for speech in adolescence, as well as its associations with personality dimensions pertaining to schizotypy and impulsivity. Furthermore, to explore whether recognition and source memory draw on similar developmental and heuristic processes, particularly as it pertains to verbally-presented stimuli, we also examine the effects of age and cognitive effort on recognition performance.

Given that verbal memory commonly improves during adolescence (Murre et al., 2013) we expect that older adolescents will exhibit better recognition for speech than younger adolescents. Furthermore, because overt reading involves the encoding of sensory-motor 
signals, which can be used to distinguish previously presented from new stimuli, we hypothesize that participants will show better recognition for overtly-read than silently-read items. Regarding self-monitoring, previous research indicates that young children perform worse than older children and adults in self-monitoring for speech (Foley et al., 1983; Sussman, 2001). Thus, we hypothesise that younger adolescents will exhibit more self-monitoring errors compared to older adolescents. In accordance with the source-monitoring framework, we also expect that our sample will demonstrate increased rates of internalizing misattributions under high cognitive effort, which generates more cognitive operations during encoding. In terms of the associations with schizotypal traits, following previous action self-monitoring research in adults (Humpston et al., 2017; Peters et al., 2007), we hypothesise that the rate of externalizing misattributions for speech will be positively associated with the cognitive-perceptual dimension of schizotypy. Given that the majority of self-monitoring studies have focused on cognitive-perceptual manifestations and no study to date has examined self-monitoring for speech and it's links to schizotypal trait expression in community adolescents, we also seek to explore whether higher scores across different schizotypy dimensions, including the interpersonal and disorganization dimensions, will be associated with increases in the total rate of self-monitoring misattributions (used in the current study as a measure of general selfmonitoring performance). Finally, on the basis of previous work involving incarcerated youths (Morosan et al., 2018) we expect that impulsivity dimensions will show positive associations with externalizing misattributions and negative associations with internalizing misattributions.

\section{Methods}

\subsection{Participants}

139 community adolescents were recruited through written advertisements in public schools in the city of Geneva, Switzerland. Inclusion criteria were age (12-18 years) and fluency in 
French. Participants were screened for cognitive impairment. Those with scores below a standard score of 7 in the Block Design subtest of the Wechsler Intelligence Scale (Wechsler, 1955) were excluded from the analyses $(n=17)$. To account for insufficient attention to the self-monitoring task, participants scoring three standard deviations below the mean $d$ ' score in the recognition test were also excluded $(n=1)$. None of the participants scored three standard deviations above the mean $d$ ' score. The final sample consisted of 121 adolescents ( 56 females, 65 males, $\left.\mathrm{M}_{\mathrm{age}}=15.23\right)$. None of the participants suffered from past/present neurological/neurogenetic disorders. Written informed consent was obtained from all participants and legal guardians.

\subsection{Measures}

\section{Self-monitoring task}

Self-monitoring was investigated using a speech-monitoring task (Debbané et al., 2010) consisting of two parts: a reading procedure, followed by a recognition and self-monitoring test. In the first phase participants were required to read, either aloud or silently, a series of common words (low cognitive effort) or non-words (high cognitive effort) presented on a computer screen. The inclusion of non-word items in the high-cognitive effort condition was based on neuroimaging research on language processing, suggesting that the reading of nonwords, which contain unfamiliar phonological associations and no immediate semantic associations, entails more effortful processing and stronger neural activation than that of words (Price \& Devlin, 2011; Taylor et al., 2013).

During the reading procedure, six blocks (three silent, three aloud) of eight items (four words, four non-words) were randomly presented. Each condition contained 12 items, for a total of 48 items (12 word/overt items, 12 non-word/overt items, 12 words/silent items and 12 non-word/silent items). After a 15-minute filler task, a recognition sheet was handed out, 
containing 72 items (48 original items,12 new word and 12 new non-word items). In the test phase, participants had to indicate if each item from the list appeared in the first part of the task (recognition test), and attribute recognized items to a reading condition, silent or aloud (selfmonitoring test). The task yields two types of self-monitoring errors: externalizing errors (attributing silently-read items as overtly-read), and internalizing errors (attributing overtlyread items as silently-read); for two item types involving different cognitive effort levels: high cognitive effort (non-words) and low cognitive effort (words).

For the recognition test, signal detection theory (Stanislaw \& Todorov, 1999) was used to assess the sensitivity for each type of items (word and non-words) for both reading conditions (aloud and silently). To estimate $d$ ' scores the $z$ score corresponding to false alarms was subtracted from the $z$ score corresponding to hits.

The percentage of externalizing misattributions was calculated by dividing the total score for items read silently but identified as read aloud in the monitoring test, out of the total number of correctly recognized silent items. The percentage of internalizing misattributions was calculated by dividing the total score of items read overtly but identified as read silently in the monitoring test, out of the total number of correctly recognized overt items. Percentages of externalizing and internalizing errors were calculated overall, as well as for each item type. Finally, the total percentage of self-monitoring errors was calculated as the sum of internalizing and externalizing errors for all items.

\section{Self-report measures}

The Schizotypal Personality Questionnaire (SPQ-French version, Dumas et al., 2000) measures schizotypal traits and their dimensions, yielding three factor scores: cognitiveperceptual (unusual perceptual experiences, suspiciousness, ideas of reference); interpersonal (social anxiety, constricted affect, lack of close friends); and disorganization (odd speech, odd behaviour). The French version of the SPQ has shown good reliability (Cronbach's alpha = 
0.91) (Dumas et al., 2000) and has been validated for use with francophone adolescents (Badoud et al., 2011).

The UPPS Impulsive Behaviour Scale - short version (UPPS-P, Billieux et al., 2012) measures four personality factors associated with impulsive behaviour: lack of premeditation (tendency to act without thinking); lack of perseverance (inability to remain focused in a task); urgency (tendency to act rashly under negative emotions); and sensation seeking (tendency to seek out novel and thrilling experiences). The UPPS-P has been validated for French-speaking samples, showing good reliability (Cronbach's alpha $=0.70-0.84)$ (Billieux et al., 2012).

\subsection{Statistical analysis}

For the self-monitoring task, a $2 \times 2 \times 3$ mixed analysis of variance (ANOVA) was conducted on the $d$ ' recognition scores with two within subject factors (item type: word vs. non-word; and reading type: aloud vs. silently), and age-group as the between factor [12-14 (early adolescents, $\mathrm{n}=36$ ), 14-16 (middle adolescents, $\mathrm{n}=39$ ), 16-18 (late adolescents, $\mathrm{n}=46$ )]. A $2 \times 2 \times 3$ mixed ANOVA was also conducted on self-monitoring error scores with two within subject factors: (monitoring error type: externalizing vs. internalizing; and item type: word vs. non-word), and age-group as the between factor [12-14 (early adolescents), 14-16 (middle adolescents), 16-18 (late adolescents)]. Because male and female participants did not differ in self-monitoring scores, gender was not entered as a covariate in these analyses.

Because all SPQ and UPPS-P dimension scores violated the assumption of normality (Kolmogorov-Smirnov test, $p<0.05$ ), Spearman's correlation coefficients were calculated to estimate their relationships with self-monitoring errors. To account for multiple comparisons, Bonferroni adjusted significance levels were calculated.

\section{Results}




\subsection{Descriptive results}

Table 1 presents the descriptive results for the variables included in our analyses.

\subsection{Self-monitoring task results}

\section{Recognition performance}

Table 2 presents the age-group means and standard deviations for the $d$ ' scores, for each type of item in both reading conditions. Results of the mixed ANOVA conducted on the $d$ ' recognition scores, with age-group as a between factor, reveal a main effect of age-group ( $F$ $(2,118)=6.23, p=0.003)$. Post-hoc pairwise comparisons suggest that independently of reading condition and item type, the early adolescent group displayed significantly less accurate recognition scores compared to middle $(p=0.02)$ and late adolescents $(p=0.001)$. In addition, a significant interaction-effect was found between reading type and age-group $(F(2$, 118) $=5.34, p=0.01$ ), suggesting that independently of item type, early adolescents less accurately recognized overtly-read items compared to middle $(p=0.004)$ and late adolescents $(p<0.001)$.

The mixed ANOVA conducted on d' scores also revealed a significant main effect of reading type $(F(1,118)=85.42, p<0.001)$, suggesting that independently of age-group and item type, silently read items are less accurately recognized. Furthermore, results showed a significant interaction effect between reading type and item type $(F(1,118)=20.30, p<$ 0.001), suggesting that independently of age-group, overtly-read words were significantly more accurately recognized than overtly-read non-words $(F(1,118)=11.70, p=0.001)$, while silently-read non-words were significantly more accurately recognized than silently-read words $(F(1,118)=4.07, p=0.046)($ Figure 1$)$. 


\section{Self-monitoring performance}

Table 3 presents the age-group means and standard deviations for the self-monitoring scores. Results of the mixed ANOVA conducted on the monitoring error scores for each type of item (words vs. non-words), with age-group as the between factor, revealed a main effect of monitoring error type $(F(1,118)=8.53, p=0.004)$, indicating a greater rate of internalizing than externalizing errors, independently of item type and age-group. Although no main effect for item type was found, the results demonstrate a significant interaction effect between monitoring error and item type $(F(1,118)=10.36, p=0.002)$, suggesting that the rate of monitoring errors affects differently each type of items. To follow-up this interaction effect, simple effects were analysed, showing a greater rate of internalizing errors for non-words than words $(F(1,118)=12.58, p=0.001)($ Figure 2$)$.

\subsection{Associations between self-monitoring errors, schizotypy and impulsivity dimensions}

Table 4 presents the correlations between all studied variables. Contrary to our hypothesis the cognitive-perceptual dimension of schizotypy was not associated with the rate of externalizing monitoring errors. Following Bonferroni correction, a significant positive correlation was found between the interpersonal SPQ dimension and the total percentage of self-monitoring errors $(r s(121)=0.22, p<0.05)$. Neither the cognitive-perceptual nor the disorganization dimensions of schizotypy were associated with the total rate of self-monitoring errors in our sample.

Regarding impulsivity, none of the UPPS-P dimensions were associated with externalizing monitoring errors. Although the "urgency" UPPS-P dimension showed a negative correlation with internalizing monitoring errors $(r s(121)=-0.22, p<0.05)$, this relationship was not significant after Bonferroni correction was applied. 


\section{Discussion}

Our study assessed the effects of age, cognitive effort and personality dimensions pertaining to schizotypy and impulsivity on self-monitoring for speech in community adolescents. We employed a task that yields a recognition score and two self-monitoring error scores (internalizing and externalizing) for two types of items involving different levels of cognitive effort (words: low cognitive effort; non-words: high cognitive effort).

\subsection{Effects of age and cognitive effort on recognition performance}

First, an age-group difference was found for recognition, suggesting that the early-adolescent group displayed worse recognition scores compared to middle and late adolescents. This is in line with developmental studies in adolescence, suggesting that memory for recognition improves with age (Murre et al., 2013). Closer inspection showed that the specific age-effect was particularly pronounced for overtly-read items. This may suggest that younger adolescents do not utilize as effectively as their older peers the sensory-motor information that accompanies overt reading (i.e. articulation, sounds), which during retrieval can help discriminate between previously presented and new items.

Furthermore in accordance with our hypothesis, we found that silently-read items were less accurately recognized compared to overtly-read items. According to Johnson et al. (1993) the lack of sensory-motor signals during memory encoding for silently-read items may deem them less distinctive than overtly-read items, thus less accurately recognized. Interestingly, the type of reading affected differently the recognition scores for each type of item. Under overt reading, word items that require low cognitive effort were more accurately recognized than high cognitive effort non-word items. Conversely, under silent reading, participants displayed better recognition for non-word than word items. These findings are in line with the sourcemonitoring framework, which states that both recognition and source memory are influenced by changes in sensory-motor precision and cognitive activity during encoding (Johnson, 2006). 
The finding that under overt reading, adolescents recognize better word than non-word items may indicate that the encoding of sensory-motor signals yields a recognition advantage for low cognitive effort material. Accordingly, better recognition for non-word than word items under silent reading suggests that the absence of sensory-motor cues during encoding generates a memory advantage for high cognitive effort material.

\subsection{Effects of age and cognitive effort on self-monitoring performance}

Contrary to our hypothesis and also in contrast to recognition performance, the present analyses failed to show an age-group effect for self-monitoring in adolescence. Indeed, research suggests that source memory is separate from recognition memory, and the two follow different developmental trajectories (Foley et al., 1983; Sussman, 2001). One possibility is that the capacity to distinguish between silent and overt speech is established prior to adolescence (Sussman, 2001). Another possibility is that our task is not sensitive enough to capture the developmental maturation that occurs in self-monitoring during adolescence (Lagioia et al., 2011).

Overall, results indicate that non-clinical youths are more prone to confuse overt speech as silent, while cognitive effort contributes to the overall rate of self-monitoring misattributions. Importantly, our sample presented a specific pattern of self-monitoring errors for high cognitive effort items. In line with our hypothesis, non-word items led to more internalizing misattributions (confusing overtly-read items as silently-read). This is consistent with the source-monitoring framework, which states that conditions that increase the cognitive effort for the encoding of overt speech also increase its similarity to silent speech, thus making it difficult to discriminate between the two (Johnson et al., 1993). Another possibility is that increased cognitive effort reduces the encoding of sensory-motor cues that typically accompany overt speech, thus engendering uncertainty about the "realness" of overtly-read items (Morosan et al., 2018). While schizophrenia sufferers (Bentall et al., 1991) and high risk 
subjects (Debbané et al., 2010; Larøi et al., 2004) display externalizing source-monitoring misattributions under high cognitive effort, typically developing adolescents demonstrate the opposite pattern.

\subsection{Associations of self-monitoring errors with personality traits of schizotypy and impulsivity}

The present analyses suggest that self-monitoring errors are not associated with cognitiveperceptual or disorganization manifestations of schizotypy in adolescence. However, a significant positive association was found between the interpersonal dimension of schizotypy and the total rate of self-monitoring errors.

The lack of association between positive schizotypy and self-monitoring was unexpected. Most studies that have examined the relationship between schizotypy and selfmonitoring, albeit in adult samples, have reported associations with positive manifestations (Humpston et al., 2017; Peters et al., 2007). However, contrary to the current study that focused on speech-monitoring, self-monitoring studies in schizotypy have primarily used actionmonitoring tasks (Humpston et al., 2017; Peters et al., 2007). Given that kinaesthetic actions entail richer sensory-motor and spatiotemporal traces than those involved in the production of overt speech, action-monitoring may constitute a relatively easier process than speechmonitoring (Collignon et al., 2005). Therefore, action-monitoring paradigms may be more sensitive than speech-monitoring tasks in capturing associations with cognitive-perceptual manifestations. Indeed, positive schizotypy has been consistently linked with difficulties in the monitoring of sensory-motor signals for both self-generated and other-produced physical actions (Itaguchi et al., 2018; Lemaitre et al., 2016). Furthermore, the only study that assessed both action and speech-monitoring in a sample of healthy adults, reported that positive schizotypy was associated only with the former (Humpston et al., 2017). 
The association between the interpersonal dimension of schizotypy and the total rate of self-monitoring misattributions in adolescence is a novel finding. Most source-monitoring studies in non-clinical samples have focused on cognitive-perceptual manifestations, either by using measures that assess hallucination and delusion-like phenomena, or by undertaking group comparisons according to the presence of these symptoms (Peters et al., 2007; Collignon et al., 2005). Contrary to these, Sahakyan and Kwapil (2016) assessed source-monitoring for verbally-presented material in a sample of healthy adults by comparing high scorers in positive schizotypy to those scoring high in negative schizotypy. They reported that the negative schizotypy subgroup exhibited significantly more source-monitoring errors compared to the positive schizotypy subgroup, indicating that negative rather than positive schizotypy involves impairments in source memory for verbally-presented material (Sahakyan \& Kwapil, 2016). Our findings add to this literature, suggesting that the link between negative schizotypy and source-monitoring errors may already be present during adolescence. One possibility is that impaired self-monitoring interferes with young people's ability to function within social situations, leading them to withdraw from interpersonal contact. Alternatively, because higherorder cognitive processes commonly develop within interpersonal relationships (Fonagy et al., 2002), reduced interpersonal contact in the context of negative schizotypy may contribute to undermine the elaboration of self-monitoring during adolescence (Debbané \& Barrantes-Vidal, 2014). Given that negative schizotypy is one of the strongest distal predictors of clinical psychosis (Debbané et al., 2014), future investigations should examine whether exacerbations of self-monitoring misattributions in the context of negative schizotypy represent markers of psychosis vulnerability.

Regarding impulsivity, no associations were found between UPPS-P facets and selfmonitoring. It has been argued that impairments in self-monitoring may contribute to limit incarcerated adolescent's insight about the negative consequences of their behaviours 
(Morosan et al. 2018). The lack of associations between self-monitoring and impulsivity in the current study suggests that this is not so for typically developing youths. Importantly however, self-report measures of impulsivity do not always correlate with behavioural assessments of impulse control, measured via response-inhibition tasks (Wilbertz et al., 2014). Thus, despite the lack of association with trait impulsivity, future studies can examine whether selfmonitoring errors in adolescence are linked to momentary difficulties in inhibitory control.

\subsection{Limitations and conclusions}

The results of the current study should be interpreted in light of certain limitations. First, the data were derived from a relatively small sample and further associations could emerge with a larger sample. Second, the analyses performed were cross-sectional, and longitudinal investigations are needed to establish causal links between negative schizotypy and selfmonitoring. Finally, previous research indicates that the emotional valance and semantic processing of word items may play a part in the association between externalizing selfmonitoring misattributions and hallucination-like experiences (Larøi et al., 2004; Sugimori et al., 2011). However, the current study did not address these factors.

Despite these limitations, this study is the first to examine self-monitoring for speech in typical adolescents and the nature of its associations with personality traits pertaining to schizotypy and impulsivity. The present analyses highlight the importance of cognitive effort and negative schizotypy on the level of self-monitoring confusions across adolescence. From an early prevention standpoint, findings suggest that self-monitoring misattributions may represent psychotherapeutic targets to attenuate trait-risk for schizophrenia in youths presenting with negative schizotypal features. 


\section{References}

Allen, P., Aleman, A., \& Mcguire, P.K. (2007). Inner speech models of auditory verbal hallucinations: evidence from behavioural and neuroimaging studies. International Review of Psychiatry, 19(4), 407-415.

Badoud, D., Billieux, J., Eliez, S., Imhof, A., Heller, P., Eytan, A., \& Debbané, M. (2015). Covariance and specificity in adolescent schizotypal and borderline trait expression. Early Intervention in Psychiatry, 9(5), 378-387.

Badoud, D., Chanal, J., der Linden Van, M., Eliez, S., \& Debbané, M. (2011). Validation study of the French schizotypal personality questionnaire in a sample of adolescents: a confirmatory factor analysis. L'encéphale, 37(4), 299-307.

Bentall, R.P., Baker, G.A., \& Havers, S. (1991). Reality monitoring and psychotic hallucinations. British Journal of Clinical Psychology, 30(3), 213-222.

Billieux, J., Rochat, L., Ceschi, G., Carré, A., Offerlin-Meyer, I., Defeldre, A.-C., Khazaal, Y., Besche-Richard, C., \& Van der Linden, M. (2012). Validation of a short French version of the UPPS-P Impulsive Behavior Scale. Comprehensive Psychiatry, 53(5), 609-615.

Blakemore, S.-J., Oakley, D.A., \& Frith, C. (2003). Delusions of alien control in the normal brain. Neuropsychologia, 41(8), 1058-1067.

Brébion, G., Amador, X., David, A., Malaspina, D., Sharif, Z., \& Gorman, J.M. (2000). Positive symptomatology and source-monitoring failure in schizophrenia — an analysis of symptom-specific effects. Psychiatry research, 95(2), 119-131.

Collignon, O., Van der Linden, M., \& Larøi, F. (2005). Source monitoring for actions in hallucination proneness. Cognitive Neuropsychiatry, 10(2), 105-123.

Debbané, M., \& Barrantes-Vidal, N. (2014). Schizotypy from a developmental perspective. Schizophrenia bulletin, 41(suppl_2), S386-S395. 
Debbané, M., Eliez, S., Badoud, D., Conus, P., Flückiger, R., \& Schultze-Lutter, F. (2014). Developing psychosis and its risk states through the lens of schizotypy. Schizophrenia bulletin, 41(suppl_2), S396-S407.

Debbané, M., Van der Linden, M., Glaser, B., \& Eliez, S. (2010). Monitoring of self-generated speech in adolescents with 22q11. 2 deletion syndrome. British Journal of Clinical Psychology, 49(3), 373-386.

Dewhurst, S.A. (1999). Cognitive effort and recollective experience in recognition memory. Memory, 7(2), 129-146.

Docherty, N.M. (2012). Missing referents, psychotic symptoms, and discriminating the internal from the externalized. Journal of abnormal psychology, 121(2), 416.

Dumas, P., Bouafia, S., Gutknecht, C., Saoud, M., Dalery, J., \& d'Amato, T. (2000). Validation of the French version of the Raine Schizotypal Personality Disorder Questionnaire. L'encéphale, 26(5), 23-29.

Ferchiou, A., Schürhoff, F., Bulzacka, E., Mahbouli, M., Leboyer, M., \& Szöke, A. (2010). Source monitoring: general presentation and review of literature in schizophrenia. L'encéphale, 36(4), 326-333.

Finke, R.A., Johnson, M.K., Shyi, G.C.-W. (1988). Memory confusions for real and imagined completions of symmetrical visual patterns. Memory \& Cognition, 16(2), 133-137.

Foley, M.A., Johnson, M.K., \& Raye, C.L. (1983). Age-related changes in confusion between memories for thoughts and memories for speech. Child development, 51-60.

Fonagy, P., Gergely, G., Jurist, E.L., \& Target, M. (2002). Affect regulation, mentalization and the development of the self: Routledge.

Fonseca-Pedrero, E., Debbané, M., Ortuño-Sierra, J., Chan, R., Cicero, D., Zhang, L., Brenner, C., Barkus, E., Linscott, R., \& Kwapil, T. (2018). The structure of schizotypal personality traits: a cross-national study. Psychological medicine, 48(3), 451-462. 
Franck, N., Rouby, P., Daprati, E., Daléry, J., Marie-Cardine, M., \& Georgieff, N. (2000). Confusion between silent and overt reading in schizophrenia. Schizophrenia Research, 41(2), 357-364.

Frith, C.D., Blakemore, S.-J., \& Wolpert, D.M. (2000). Explaining the symptoms of schizophrenia: abnormalities in the awareness of action. Brain Research Reviews, 31(23), 357-363.

Gawęda, Ł., Moritz, S., \& Kokoszka, A. (2012). Impaired discrimination between imagined and performed actions in schizophrenia. Psychiatry research, 195(1-2), 1-8.

Gooding, D.C., Tallent, K.A., \& Matts, C.W. (2005). Clinical status of at-risk individuals 5 years later: further validation of the psychometric high-risk strategy. Journal of abnormal psychology, 114(1), 170.

Henquet, C., Krabbendam, L., Dautzenberg, J., Jolles, J., \& Merckelbach, H. (2005). Confusing thoughts and speech: source monitoring and psychosis. Psychiatry research, 133(1), $57-63$.

Humpston, C.S., Linden, D.E., \& Evans, L.H. (2017). Deficits in reality and internal source monitoring of actions are associated with the positive dimension of schizotypy. Psychiatry research, 250, 44-49.

Itaguchi, Y., Sugimori, E., \& Fukuzawa, K. (2018). Schizotypal traits and forearm motor control against self-other produced action in a bimanual unloading task. Neuropsychologia, 113, 43-51.

Johns, L., Allen, P., Valli, I., Winton-Brown, T., Broome, M., Woolley, J., Tabraham, P., Day, F., Howes, O., \& Wykes, T. (2010). Impaired verbal self-monitoring in individuals at high risk of psychosis. Psychological medicine, 40(9), 1433-1442. 
Johns, L.C., Gregg, L., Allen, P., \& McGUIRE, P.K. (2006). Impaired verbal self-monitoring in psychosis: effects of state, trait and diagnosis. Psychological medicine, 36(4), 465474.

Johnson, M.K., Hashtroudi, S., \& Lindsay, D.S. (1993). Source monitoring. Psychological bulletin, 114(1), 3 .

Johnson, M.K. (2006). Memory and reality. American Pysychologist, 61(8), 760.

Johnson, M.K. (1988). Reality monitoring: An experimental phenomenological approach. Journal of Experimental Psychology, 117(4), 390.

Keefe, R.S., Arnold, M., Bayen, U., \& Harvey, P. (1999). Source monitoring deficits in patients with schizophrenia; a multinomial modelling analysis. Psychological medicine, 29(4), 903-914.

Lagioia, A., Eliez, S., Schneider, M., Simons, J.S., Van der Linden, M., \& Debbané, M. (2011). Neural correlates of reality monitoring during adolescence. NeuroImage, 55(3), 13931400.

Larøi, F., Van der Linden, M., \& Marczewski, P. (2004). The effects of emotional salience, cognitive effort and meta-cognitive beliefs on a reality monitoring task in hallucinationprone subjects. British Journal of Clinical Psychology, 43(3), 221-233.

Lemaitre, A.-L., Luyat, M., Lafargue, G. (2016). Individuals with pronounced schizotypal traits are particularly successful in tickling themselves. Consciousness and Cognition, $41,64-71$.

Moeller, F.G., Barratt, E.S., Dougherty, D.M., Schmitz, J.M., \& Swann, A.C. (2001). Psychiatric aspects of impulsivity. American Journal of Psychiatry, 158(11), 17831793. 
Morosan, L., Badoud, D., Salaminios, G., Eliez, S., Van der Linden, M., Heller, P., \& Debbané, M. (2018). Patterns of source monitoring bias in incarcerated youths with and without conduct problems. Cognitive Neuropsychiatry, 23(1), 15-27.

Müller, V.I., Langner, R., Cieslik, E.C., Rottschy, C., Eickhoff, S.B. (2015). Interindividual differences in cognitive flexibility: influence of gray matter volume, functional connectivity and trait impulsivity. Brain Structure and Function, 220(4), 2401-2414.

Murre, J.M., Janssen, S.M., Rouw, R., \& Meeter, M.J. (2013). The rise and fall of immediate and delayed memory for verbal and visuospatial information from late childhood to late adulthood. Acta Psychologica, 142(1), 96-107.

Peters, M.J., Smeets, T., Giesbrecht, T., Jelicic, M., \& Merckelbach, H. (2007). Confusing action and imagination: action source monitoring in individuals with schizotypal traits. The Journal of nervous and mental disease, 195(9), 752-757.

Price, C.J., \& Devlin, J.T. (2011). The interactive account of ventral occipitotemporal contributions to reading. Trends in Cognitive Sciences, 15(6), 246-253.

Raine, A. (2006). Schizotypal personality: neurodevelopmental and psychosocial trajectories. Annu. Rev. Clin. Psychol., 2, 291-326.

Sahakyan, L., \& Kwapil, T.R. (2016). Positive schizotypy and negative schizotypy are associated with differential patterns of episodic memory impairment. Schizophrenia Research: Cognition, 5, 35-40.

Sugimori, E., Asai, T., Tanno, Y. (2011). Sense of agency over thought: external misattribution of thought in a memory task and proneness to auditory hallucination. Consciousness and Cognition, 20(3), 688-695.

Sussman, A.L. (2001). Reality monitoring of performed and imagined interactive events: Developmental and contextual effects. Journal of Experimental Child Psychology, 79(2), 115-138. 
Taylor, J., Rastle, K., \& Davis, M.H. (2013). Can cognitive models explain brain activation during word and pseudoword reading? A meta-analysis of 36 neuroimaging studies. Psychological Bulletin, 139(4), 766.

Wechsler, D. (1955). Manual for the Wechsler adult intelligence scale. The Psychological Corporation. New York.

Whiteside, S.P., \& Lynam, D.R. (2001). The five factor model and impulsivity: Using a structural model of personality to understand impulsivity. Personality and Individual Differences, 30(4), 669-689.

Wilbertz, T., Deserno, L., Horstmann, A., Neumann, J., Villringer, A., Heinze, H.-J., Boehler, C.N., \& Schlagenhauf, F. (2014). Response inhibition and its relation to multidimensional impulsivity. Neuroimage, 103, 241-248. 
Table 1. Means and standard deviations for each variable in the sample

\begin{tabular}{lcccc}
\hline & Mean & SD & Minimum & Maximum \\
\hline Recognition Test & & & & \\
Correct recognition/Hits raw score & 29.37 & 5.69 & 13.00 & 44.00 \\
Correct recognition/Hits (\%) & 61.19 & 11.86 & 27.00 & 92.00 \\
False alarms raw score & 4.59 & 3.00 & 0.00 & 15.00 \\
False alarms (\%) & 19.21 & 12.41 & 0.00 & 63.00 \\
d-prime read words & 2.05 & 0.65 & 0.71 & 3.46 \\
d-prime silent words & 1.33 & 0.53 & 0.24 & 1.33 \\
d-prime read non-words & 1.79 & 0.64 & 0.00 & 3.46 \\
d-prime silent non-words & 1.47 & 0.61 & 0.21 & 3.11 \\
\hline Self-monitoring Test & & & & \\
Self-monitoring errors Total (\%) & 32.39 & 11.35 & 0.00 & 56.88 \\
Internalizing errors Total (\%) & 34.83 & 14.48 & 0.00 & 68.18 \\
Externalizing errors Total (\%) & 29.95 & 15.54 & 0.00 & 70.00 \\
Internalizing errors for words (\%) & 30.28 & 19.30 & 0.00 & 30.28 \\
Internalizing errors for non-words (\%) & 39.40 & 21.40 & 0.00 & 100.00 \\
Externalizing errors for words (\%) & 32.24 & 22.90 & 0.00 & 100.00 \\
Externalizing errors for non-words (\%) & 27.66 & 20.24 & 0.00 & 83.33 \\
\hline Schizotypal Traits (SPQ) & & & & \\
SPQ Cognitive-perceptual dimension & 8.58 & 6.83 & 0.00 & 33.00 \\
SPQ Interpersonal dimension & 6.63 & 4.54 & 0.00 & 20.00 \\
SPQ Disorganization dimension & 5.45 & 3.93 & 0.00 & 14.00 \\
\hline Impulsivity (UPPS-P) & & & & \\
UPPS Lack of Perseverance & 2.04 & 0.60 & 1.00 & 4.00 \\
UPPS Lack of Premeditation & 2.17 & 0.61 & 1.00 & 4.00 \\
UPPS Urgency & 2.46 & 0.68 & 1.00 & 4.00 \\
UPPS Sensation Seeking & 2.69 & 0.69 & 1.00 & 4.00 \\
\hline & & & & \\
\hline
\end{tabular}

Note: SPQ, Schizotypal Personality Questionnaire; UPPS, Impulsive Behaviour Scale 
Table 2. Age-group means and standard deviations for Hits, false alarm and d-prime scores

\begin{tabular}{|c|c|c|c|c|c|c|}
\hline \multirow[b]{2}{*}{ Recognition Test } & \multicolumn{2}{|c|}{$\begin{array}{c}\text { Early Adolescents } \\
(\mathrm{n}=36, \text { age }= \\
13.04)\end{array}$} & \multicolumn{2}{|c|}{$\begin{array}{l}\text { Middle Adolescents } \\
(\mathrm{n}=39, \text { age }=15.13)\end{array}$} & \multicolumn{2}{|c|}{$\begin{array}{c}\text { Late adolescents } \\
(\mathrm{n}=46, \text { age }=17.02)\end{array}$} \\
\hline & Mean & SD & Mean & SD & Mean & SD \\
\hline $\begin{array}{l}\text { Correct recognition/Hits raw } \\
\text { score }\end{array}$ & 27.69 & 5.37 & 28.85 & 5.05 & 31.13 & 6.06 \\
\hline Correct recognition/Hits (\%) & 57.70 & 11.19 & 60.10 & 10.52 & 64.86 & 12.64 \\
\hline False alarms raw score & 5.11 & 3.19 & 3.92 & 2.64 & 4.76 & 3.10 \\
\hline False alarms $(\%)$ & 21.30 & 13.28 & 16.35 & 11.00 & 19.84 & 12.91 \\
\hline d-prime Total & 1.51 & 0.29 & 1.68 & 0.30 & 1.76 & 0.36 \\
\hline d-prime read words & 1.79 & 0.67 & 2.10 & 0.56 & 2.20 & 0.64 \\
\hline d-prime silent words & 1.35 & 0.53 & 1.28 & 0.56 & 1.35 & 0.51 \\
\hline d-prime read non-words & 1.50 & 0.63 & 1.80 & 0.65 & 2.02 & 0.55 \\
\hline d-prime silent non-words & 1.40 & 0.56 & 1.53 & 0.60 & 1.47 & 0.66 \\
\hline
\end{tabular}


Table 3. Age-group means and standard deviations for self-monitoring error percentages

\begin{tabular}{|c|c|c|c|c|c|c|}
\hline \multirow[b]{2}{*}{ Self-monitoring Test } & \multicolumn{2}{|c|}{$\begin{array}{c}\text { Early Adolescents } \\
(\mathrm{n}=36, \text { age }= \\
13.04)\end{array}$} & \multicolumn{2}{|c|}{$\begin{array}{l}\text { Middle Adolescents } \\
(\mathrm{n}=39, \text { age }=15.13)\end{array}$} & \multicolumn{2}{|c|}{$\begin{array}{c}\text { Late adolescents } \\
(\mathrm{n}=46, \text { age }=17.02)\end{array}$} \\
\hline & Mean & SD & Mean & SD & Mean & SD \\
\hline Self-monitoring errors Total (\%) & 32.83 & 10.22 & 31.01 & 11.79 & 33.22 & 11.92 \\
\hline Internalizing errors Total (\%) & 37.06 & 17.15 & 34.26 & 13.50 & 33.61 & 13.06 \\
\hline Externalizing errors Total (\%) & 28.59 & 13.63 & 27.81 & 15.54 & 32.82 & 16.79 \\
\hline $\begin{array}{l}\text { Internalizing errors for words } \\
(\%)\end{array}$ & 32.90 & 21.82 & 27.22 & 18.34 & 30.81 & 18.00 \\
\hline $\begin{array}{l}\text { Internalizing errors for non- } \\
\text { words }(\%)\end{array}$ & 41.22 & 25.86 & 41.19 & 16.77 & 36.42 & 21.19 \\
\hline $\begin{array}{l}\text { Externalizing errors for words } \\
(\%)\end{array}$ & 31.05 & 19.94 & 28.75 & 23.78 & 36.11 & 23.78 \\
\hline $\begin{array}{l}\text { Externalizing errors for non- } \\
\text { words }(\%)\end{array}$ & 26.13 & 18.80 & 26.87 & 21.74 & 29.52 & 20.31 \\
\hline
\end{tabular}

Table 4. Correlations between self-monitoring error percentages, SPQ and UPPS-P dimensions (before Bonferroni correction applied)

\begin{tabular}{|c|c|c|c|c|c|c|c|c|c|c|c|}
\hline & & 1 & 2 & 3 & 4 & 5 & 6 & 7 & 8 & 9 & 10 \\
\hline 1. & Self-Monitoring Errors Total (\%) & - & $0.78^{* *}$ & $0.74^{* *}$ & -0.01 & $0.22^{*}$ & 0.03 & -0.11 & -0.09 & -0.09 & 0.01 \\
\hline 2. & Externalizing Monitoring Errors (\%) & & - & 0.14 & 0.09 & 0.17 & 0.07 & -0.04 & -0.13 & 0.05 & 0.07 \\
\hline 3. & Internalizing Monitoring Errors (\%) & & & - & -0.01 & 0.11 & -0.06 & -0.12 & -0.02 & $-0.22^{*}$ & -0.12 \\
\hline 4. & SPQ Cognitive-Perceptual & & & & - & $0.56^{* *}$ & $0.70^{* *}$ & -0.02 & 0.07 & $0.34^{* *}$ & $0.20^{*}$ \\
\hline 5. & SPQ Interpersonal & & & & & - & $0.53^{* *}$ & -0.16 & -0.04 & $0.33^{* *}$ & 0.06 \\
\hline 6. & SPQ Disorganization & & & & & & - & 0.13 & 0.04 & $0.34^{* *}$ & $0.22^{*}$ \\
\hline 7. & UPPS Lack of Premeditation & & & & & & & - & $0.38^{* *}$ & 0.02 & 0.08 \\
\hline 8. & UPPS Lack of Perseverance & & & & & & & & - & -0.03 & 0.07 \\
\hline & UPPS Urgency & & & & & & & & & - & $0.24^{* *}$ \\
\hline & UPPS Sensation Seeking & & & & & & & & & & - \\
\hline
\end{tabular}


